Abstract ID: 65

\title{
Functional outcome of anterior cruciate ligament (ACL) reconstruction: comparison of bone-patella tendon-bone (BPTB) vs. Hamstring graft
}

Jeffrey Jaya Raj ${ }^{\mathrm{a}}$ | Nik Mohd Fatmy Nik Mohd Najmi ${ }^{\mathrm{b}} \mid$ Mohammad Amirrudin Hamdan $^{\mathrm{c}}$ | Aminudin Che Ahmad $^{\mathrm{b}}$ | Ahmad Hafiz Zulkifly

${ }^{a}$ Hospital Queen Elizabeth, Kota Kinabalu Sabah

${ }^{b}$ Kulliyyah of Medicine, International Islamic University Malaysia

${ }^{c}$ Hospital Angkatan Tentera Tuanku Mizan, Kuala Lumpur

Introduction: This is a cross-sectional study to evaluate the functional outcome of $A C L$ Reconstruction between BPTB and hamstring graft who underwent ACL Reconstruction between January to June 2012 at our institution. Methods: Functional IKDC scoring was done pre-operatively, at 6 months and one year. Arthrometric measurements were taken using KT2000. Lysholm score and SF-36 were assessed at one year. Resuts: All patients are male, mean age is 29 (21 to 37 years old). 21.4\% patients from patella BPTB group and 13\% of hamstring group had anterior knee pain. $50 \%$ of patients from both groups complained of numbness over operative site. Average time return to pre-injury sports activity for BPTB group was 7.9 months, whereas for hamstring group was 8.3 months. KT-2000 measurements revealed an average side to side laxity difference average of $6.6 \mathrm{~mm}$ in BPTB group and $7.9 \mathrm{~mm}$ in hamstring group. Pivot shift were negative at 6 months in all patients. IKDC knee scoring significantly improved from grade C or D to grade B in $82 \%$ of BPTB group and $63 \%$ in hamstring group one year post operatively. There were no difference between both groups in terms of SF-36, Lysholm score and knee range of motion. Conclusions: There are no difference in functional outcome and lysholm knee score between the two groups although there is a discrepancy of IKDC score and arthrometric measurement at one year. Although both groups have moderate anterior laxity after one year, but they are asymptomatic and able to go back to preinjury sports activities.

KEYWORDS: ACL reconstruction, BPTB, Hamstring 